\title{
Glioblastoma: Targeting Angiogenesis and Tyrosine Kinase Pathways
}

ISSN: 2637-773X

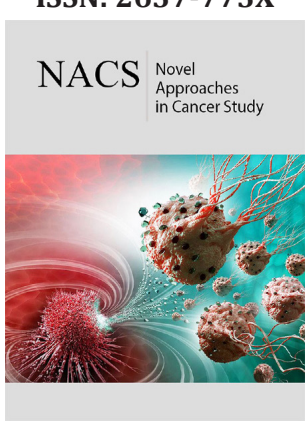

*Corresponding author: Meser M Ali, Cellular and Molecular Imaging Lab, Department of Neurosurgery, Detroit, MI, USA

Submission: 佂 May 18, 2020

Published: 漹 May 29, 2020

Volume 4 - Issue 5

How to cite this article: Ali $\mathrm{S}$ Arbab, Meser M Ali. Glioblastoma: Targeting Angiogenesis and Tyrosine Kinase Pathways. Nov Appro in Can Study. 4(5). NACS.000596. 2020. DOI: 10.31031/NACS.2020.04.000596

Copyright@ Meser M Ali. This article is distributed under the terms of the Creative Commons Attribution 4.0 International License, which permits unrestricted use and redistribution provided that the original author and source are credited.

\author{
Ali S Arbab ${ }^{1}$ and Meser M Ali ${ }^{2 *}$ \\ ${ }^{1}$ Tumor Angiogenesis Laboratory, Georgia Cancer Center, Augusta University, USA \\ ${ }^{2}$ Cellular and Molecular Imaging Lab, Department of Neurosurgery, Henry Ford Hospital, USA
}

\begin{abstract}
Angiogenesis is a hallmark of glioblastoma (GBM) and remains an important therapeutic target in its treatment, especially for recurrent GBM. GBMs are characterized by the release of vascular endothelial growth factor (VEGF), an important regulator and promoter of angiogenesis. Therefore, antiangiogenic therapies (AATs) targeting VEGF or VEGF receptors (VEGFRs) were designed and thought to be an effective tool for controlling the growth of GBM. However, recent results of different clinical trials using humanized monoclonal antibodies against VEGF (bevacizumab), as well as tyrosine kinase inhibitors (TKIs) that target different VEGFRs alone or in combination with other therapeutic agents demonstrated mixed results, with the majority of reports indicating that GBM developed resistance against antiangiogenic treatments.
\end{abstract}

Keywords: Glioblastoma; Angiogenesis; Tyrosine kinase inhibitors

\section{Introduction}

Gliomas of astrocytic, oligodendroglial, and ependymal origin account for more than $80 \%$ of malignant brain tumors. Patients with these tumors who progress to grade IV glioblastoma (GBM), the most malignant histological subtype [1], have the poorest prognosis for survival, with high potential for fatal outcome [2]. GBM constitutes about $57 \%$ of the average annual age-adjusted incidence rate of all neuroepithelial tumors and about $48 \%$ of all malignant brain and CNS tumors [3,4]. Current treatment options include surgery, radiotherapy (RT), and chemotherapy (temozolomide). Unfortunately, prognosis remains extremely poor and the median survival of 12 to 15 months in GBM with optimal treatment, including resection, radiotherapy (RT), and chemotherapy has not changed much over 3 decades. Without resection, the mean survival time continues to be 3 months. Therapeutic options are limited and must address the infiltrative nature and prominent angio- and vasculo-genesis of these aggressive tumors to develop an effective therapeutic approach with a uniform outcome for all patients [5]. Herewith, we review the current focus on therapeutic targets - angiogenesis and tyrosine kinase (TK) pathways and how these targets are manipulated in chemotherapeutic development, and other novel therapeutic approaches for the treatment of GBM.

\section{Results and Discussion}

Angiogenesis and increased vascularization are essential for the survival and proliferation of glioma cells [6]. On the other hand, malignant gliomas are characterized by the release of vascular endothelial growth factor (VEGF), an important regulator and promoter of angiogenesis [7]. The normal VEGF pathway starts when cells are lacking oxygen, which leads to the production of the hypoxia- inducible factor. This leads to releasing of VEGF followed by binding of the VEGF to VEGF receptors (VEGFRs), stimulating the tyrosine kinase pathway and ultimately resulting in angiogenesis $[8,9]$. Therefore, anti-angiogenic therapies targeting 
VEGF or VEGFRs have been designed and expected to be an effective strategy for controlling the growth of malignant gliomas. FDA approved Bevacizumab, a humanized monoclonal antibody against VEGF for recurrent GBM in 2009[10,11]. A subgroup of the patient population was benefitted when bevacizumab was combined with cytotoxic drug lomustine. Although anti-VEGF therapy has shown benefits in the reduction of vasogenic edema [9], several studies indicated that bevacizumab was not effective to improve overall survival in newly diagnosed GBM patients $[12,13]$.

Other anti-angiogenic agents have been proposed for GBM treatment, such as VEGF receptor tyrosine kinase inhibitors. However, recent results of early clinical trials using smallmolecule tyrosine kinase inhibitors (TKIs) that target different VEGFRs alone or in combination with other therapeutic agents $[7,14,15]$ demonstrated mixed results, with the majority of reports indicating that gliomas developed resistance to the employed anti-angiogenic therapies [14,16,17]. Recently, VEGF-independent tumor vascularization and resistance to Bevacizumab have also been reported for patient-derived GBM model [18]. Our work [19] in a rat orthotopic human glioma model indicated paradoxically increased production of VEGF at the peripheral part of tumors, as well as, the elevated expression of hypoxia- inducible factor-
$1 \alpha$ (HIF-1 $\alpha$ ) and SDF-1 (Figure 1). Therefore, we extended our studies using broader tyrosine kinase inhibitors that affect not only the VEGFR tyrosine kinase but also other tyrosine kinases as well. One such drug is sunitinib, a small molecule multitarget receptor tyrosine kinase inhibitor, which is known to inhibit signaling through multiple receptors such as platelet-derived growth factor receptors (PDGFRs), VEGFRs, c-KIT, colonystimulating factor-1 receptor, and fetal liver kinase 3-internal tandem duplication (FLT3-ITD). However, sunitinib treatment outcome was also not satisfactory (Figure 2). Therefore, it was thought that another probable mechanism of AAT resistance could include SDF-1 pathway-mediated mobilization of bone marrowderived endothelial progenitor cells through CXCR4 receptors on these cells. A recent study shows that depleting bone marrow cells or CXCR4 interaction can potentiate the effect of vatalanib to some extent, but the overall therapeutic benefit is not significant [20]. SDF-1 is a strong chemoattractant for CXCR4 positive cells and hindering the interaction of SDF-1/CXCR4 could be a viable mechanism to prevent vasculogenesis. Continuous treatment with AMD3100, which is a potent CXCR4 receptor antagonist, or similar CXCR4 receptor antagonists can inhibit tumor growth by preventing vasculogenesis.
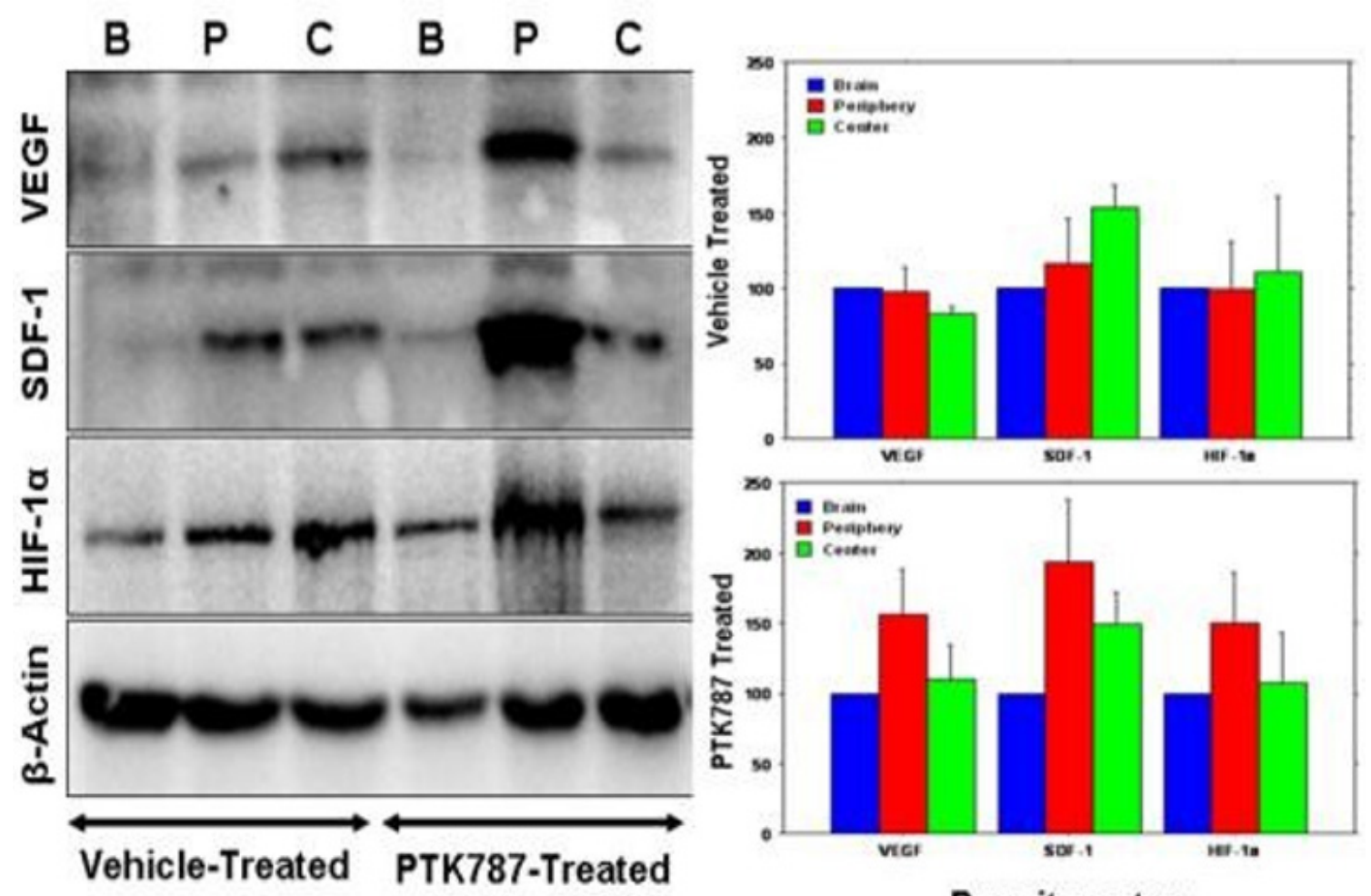

Densitometry

Figure 1: Expression of different angiogenic factors (tumors from representative cases at the peripheral (P), central part of the tumors $(\mathrm{C})$ and contralateral brains (B). Note the increased expression of VEGF, SDF-1 and HIF- 1a on western blot in tissues collected from peripheral part of the tumors, left panel) in the vehicle- and PTK787-treated part of PTK787 treated tumors. Right panel shows the densitometry analysis of the blot (\% $\beta$-actin and normalized to contralateral brain). The analysis also confirmed the finding of the blot. Ali MM, Janic B, Babajani-Feremi A, Varma

RS, Iskander ASM, et al. (2010) Changes in vascular permeability and expression of different angiogenic factors following anti-angiogenic treatment in rat glioma [19]. 


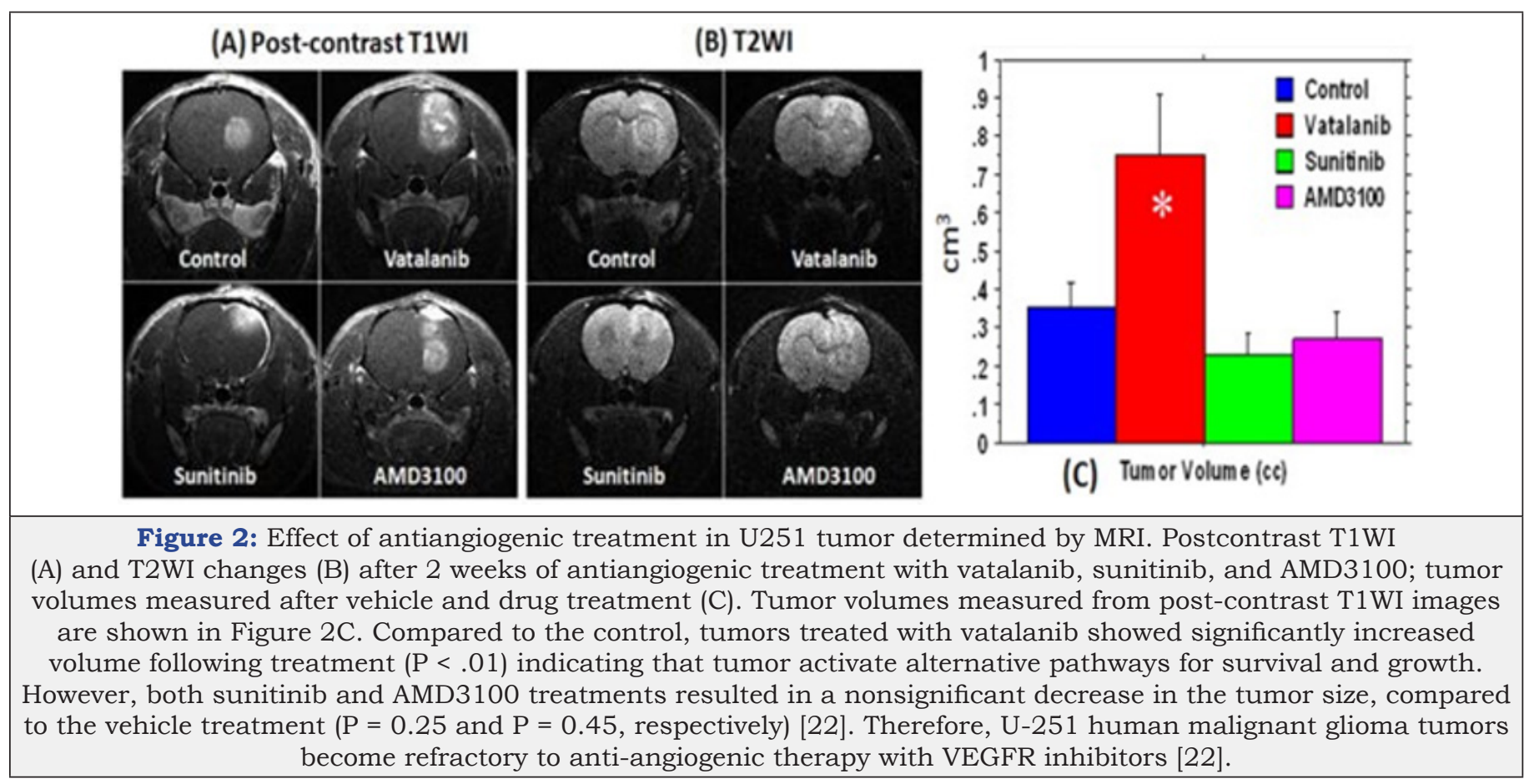

We then further examined the effects of CXCR4 antagonists and TKIs on tumor growth and angiogenesis using a rat glioma model [21]. Although, the AMD3100-treated group demonstrated moderate tumor growth inhibition, significant changes in vascular density was not observed. Sunitinib-treated animals showed significantly higher migration of the invasive cells at the peripheral part of the tumor. However, both vatalanib- and AMD3100-treated animals, the invasive cell migration distance was relatively lower compared to that of control in the tumor boundary. None of these treatment options induced necrosis at the core of the tumor. But yet, tumor growth and the blood vessel development were continued with the overexpression of a series of pro-angiogenic factors [22]. Whole-genome exon sequencing studies implicate mutations in the receptor tyrosine kinase pathways (RTK) for driving tumor growth in over $80 \%$ of GBMs. In spite of various RTKs being mutated or altered in the majority of GBMs, clinical studies have not been able to demonstrate the efficacy of molecularly targeted therapies using TKIs in GBMs [23]. In addition, other anti-angiogenic agents including an RGD peptide (Cilengitide) that binds $\alpha v \beta 3$ and $\alpha v \beta 5$ integrins upregulated on tumor neovasculature also failed in clinical trials. Therefore, angiogenesis inhibitors, which attempt to prevent new blood vessels from forming (neovascularization) and do not generally target preexisting blood vessels are not sufficient to inhibit tumor angiogenesis [24,25]. Nevertheless, both AATs and TKIs induced hypoxia [26] as a compensatory effect of the applied therapies. One of the resistance mechanisms for AATs and TKIs therapies is the induction of hypoxia and subsequent up regulation of downstream genes.

Targeting of epidermal growth factor receptor (EGFR) with small molecules or monoclonal antibodies has been reported to offer no survival benefit, [27] despite the fact that EGFR is the most common genomically altered oncogene in GBM and targeting
EGFR has shown benefit in other cancers. Multiple kinase pathways are activated in GBMs and sorafenib is a small molecule multiple kinase inhibitor that inhibits RAF, VEGFR, PDGFR, c-KIT, and FMSlike tyrosine kinase-3 targets [28]. However, a phase III clinical trial of sorafenib alone also failed recently. Alternative therapies known as tumor-vascular disrupting agents diverge from anti-angiogenic strategies and directly target established tumor vasculature. We then investigated the therapeutic effect of the tumor-vascular disrupting agent nanocombretastatin (G3-CA4) in an orthotopic glioma model with MRI monitoring. Intravenous delivery of G3CA4 across blood-brain tumor barrier (BBTB) showed a significant tumor blood flow decrease in an experimental rat model of glioma. In addition, G3-CA4 induced intratumoral blood vessels collapse leading to necrosis at the core of the tumor while the blood vessels at the periphery were alive [29].

\section{Conclusion}

A potent antiangiogenic strategy for controlling tumors has developed with endothelial cells in the tumor vasculatures as the target. The strategy can work either by delivery of drug substances inhibiting the pro-angiogenic factors that induce proliferation and migration of these cells to form blood vessels or by simply killing these cells but the latter has much greater risks if selectivity is inadequate. Inhibition of angiogenic factors such as VEGF, basic fibroblast growth factor, or their cognate receptors in endothelial cells of the growing vasculature is not sufficient to inhibit tumor angiogenesis. Both AATs and TKIs induced hypoxia [26] as a compensatory effect of the applied therapies. One of the resistance mechanisms for AATs and TKIs therapies is the induction of hypoxia and subsequent upregulation of downstream genes. Therefore, targeting hypoxia as well as hypoxia-regulated downstream genes may enhance the outcome of AATs, TKIs, and radiotherapy. 


\section{Acknowledgment}

The authors acknowledge research support from the National Institutes of Health (NIH) grant R01CA206190 to MMA.

\section{References}

1. Goodenberger ML, Jenkins RB (2012) Genetics of adult glioma. Cancer Genetics 205(12): 613-621.

2. Ohgaki H, Kleihues $P$ (2005) Epidemiology and etiology of gliomas. Acta Neuropathologica 109(1): 93-108.

3. Ostrom QT, Cioffi G, Gittleman H, Patil N, Waite K, et al. (2019) CBTRUS statistical report: Primary brain and other central nervous system tumors diagnosed in the United States in 2012-2016. Neuro Oncology 21(Suppl 5): v1-v100.

4. Ly KI, Wen PY, Huang RY (2020) Imaging of central nervous system tumors based on the 2016 World Health Organization classification. Neurol Clin 38(1): 95-113.

5. Anjum K, Shagufta BI, Abbas SQ Seema P, Ishrat K, et al. (2018) Corrigendum to "current status and future therapeutic perspectives of glioblastoma multiforme (GBM) therapy: A review" [Biomed. Pharmacother. 92: 681-689]. Biomedicine \& Pharmacotherapy 101: 820.

6. Goldbrunner RH, Bendszus M, Wood J, Kiderlen M, Sasaki M, et al. (2004) PTK787/ZK222584, an inhibitor of vascular endothelial growth factor receptor tyrosine kinases, decreases glioma growth and vascularization. Neurosurgery 55(2): 426-432.

7. Norden AD, Young GS, Setayesh K, Muzikansky A, Klufas R, et al. (2008) Bevacizumab for recurrent malignant gliomas: efficacy, toxicity, and patterns of recurrence. Neurology 70(10): 779-787.

8. Alifieris C, Trafalis DT (2015) Glioblastoma multiforme: Pathogenesis and treatment. Pharmacology \& Therapeutics 152: 63-82.

9. Rajaratnam V, Islam MM, Yang M, Slaby R, Ramirez HM, et al. (2020) Glioblastoma: Pathogenesis and current status of chemotherapy and other novel treatments. Cancers (Basel) 12(4): 937.

10. Wick W, Weller M, van den Bent M, Stupp R (2010) Bevacizumab and recurrent malignant gliomas: A European perspective. Journal of Clinical Oncology 28(12): e188-e189.

11. Agha CA, Ibrahim S, Hassan A, Elias DA, Fathallah-Shaykh HM (2010) Bevacizumab is active as a single agent against recurrent malignant gliomas. Anticancer Research 30(2): 609-611.

12. Gilbert MR, Dignam JJ, Armstrong TS, Wefel JS, Deborah TB, et al. (2014) A randomized trial of bevacizumab for newly diagnosed glioblastoma. The New England Journal of Medicine 370(8): 699-708.

13. Chinot OL, Wick W, Mason W, Henriksson R, Saran F, et al. (2014) Bevacizumab plus radiotherapy-temozolomide for newly diagnosed glioblastoma. The New England Journal of Medicine 370(8): 709-722.

14. Sierra JR, Cepero V, Giordano S (2010) Molecular mechanisms of acquired resistance to tyrosine kinase targeted therapy. Molecular Cancer 9: 75.
15. Norden AD, Drappatz J, Wen PY (2008) Novel anti-angiogenic therapies for malignant gliomas. Lancet Neurology 7(12): 1152-1160.

16. Verhoeff JJ, van Tellingen O, Claes A, Stalpers LJ, Myra E, et al. (2009) Concerns about anti-angiogenic treatment in patients with glioblastoma multiforme. BMC Cancer 9: 444.

17. Ahluwalia MS, Gladson CL (2010) Progress on antiangiogenic therapy for patients with malignant glioma. Journal of Oncology 689018.

18. Baker GJ, Yadav VN, Motsch S, Koschmann C, Calinescu A, et al. (2014) Mechanisms of glioma formation: iterative perivascular glioma growth and invasion leads to tumor progression, VEGF-independent vascularization, and resistance to antiangiogenic therapy. Neoplasia 16(7): 543-561.

19. Ali MM, Janic B, Babajani-Feremi A, Varma RS, Iskander ASM, et al. (2010) Changes in vascular permeability and expression of different angiogenic factors following anti-angiogenic treatment in rat glioma. PloS One 5(1): e8727.

20. Shaaban S, Alsulami M, Arbab SA, Ara R, Shankar A, et al. (2016) Targeting bone marrow to potentiate the anti- tumor effect of tyrosine kinase inhibitor in preclinical rat model of human glioblastoma. Int J Cancer Res 12(2): 69-81.

21.(2013) Ado-trastuzumab emtansine (Kadcyla) for HER2-positive metastatic breast cancer. The Medical Letter on Drugs and Therapeutics 55(1425): 75-76.

22. Ali MM, Kumar S, Shankar A, Ara R, Shankar A, et al. (2013) Effects of tyrosine kinase inhibitors and CXCR4 antagonist on tumor growth and angiogenesis in rat glioma model: MRI and protein analysis study. Translational Oncology 6(6): 660-669.

23. Joshi AD, Loilome W, Siu IM, Tyler B, Gallia GL, et al. (2012) Evaluation of tyrosine kinase inhibitor combinations for glioblastoma therapy. PloS One 7(10): e44372.

24. Lorusso PM, Boerner SA, Hunsberger S (2011) Clinical development of vascular disrupting agents: what lessons can we learn from $\mathrm{ASA}_{4} 0_{4}$ ? Journal of Clinical Oncology 29(22): 2952-2955.

25. Hinnen P, Eskens FA (2007) Vascular disrupting agents in clinical development. British Journal of Cancer 96(8): 1159-1165.

26.Jain RK (2014) Antiangiogenesis strategies revisited: from starving tumors to alleviating hypoxia. Cancer Cell 26(5): 605-622.

27. Rich JN, Reardon DA, Peery T, JM Dowell , JA Quinn, et al. (2004) Phase II trial of gefitinib in recurrent glioblastoma. Journal of Clinical Oncology 22(1):133-142.

28. Chang CY, Cheng TJ, Chang FR, Wang HY, Kan WC, et al. (2011) Macrophage mediated anti-proliferation effects of Anthodia camphorata non-polysaccharide based extracts on human hepatoma cells. Bioscience, Biotechnology, and Biochemistry 75(4): 624-632.

29. Gonawala S, Aryal M, Ewing JR, deCarvalho AC, Kalkanis S, Ali MM (2018) MRI monitoring of cerebral blood flow after the delivery of nanocombretastatin across the blood brain tumor barrier. J Nanomed Nanotechnol 9(5): 516. 